\section{Automatic Particle Picking From Electron Micrographs}

K. Ramani Lata, P. Penczek and J. Frank

New York State Department of Health, Albany, NY

The present-day interactive manual selection of biological molecules from digitized micrographs for single particle averaging and reconstruction requires substantial effort and time. Thus a computer algorithm capable of recognition of structural content and selection of particles would be desirable. A few approaches ${ }^{1-3}$ have been proposed in the past. The method by Frank and Wagenknecht is based on the principle of correlation search. Van Heel's method $^{2}$ is based on the computation of the local variance over a small area around each point of the image field. The method by Harauz and FongLochovsky ${ }^{3}$ is based on edge-detection. The present work was focussed on the detection and classification of particles by exploiting the standard statistical methods of discriminant analysis.

the proposed technique is described in the block diagram (Fig. 1). As illustrated in the figure, the program consists of three distinct segments devoted to, respectively, preparation of the data, training session and automatic selection based on a discriminant function set up in the training. In 1 the data preparation segment, the micrograph is (i) reduced four-fold in size, 2. (ii) low-pass filtered and (iii) run through a peak search algorithm. In the 3 . training session, the coordinates of the peak positions are used to window out 4 . small images. Manual interaction is mandatory at this point to select 5 . comparable numbers of images with particles, noise and "junk" (i.e., particle 6 . fragments, stain accumulations, etc.) from the above windowed series. Statistical parameters (i.e., average, variance, skewness, and entropy) evaluated for these selected images serve as input variables to the discriminant function routine which yields the discriminant function and

classifies the image set into the three possible categories (viz. particles, noise and "junk"). In the selection segment of the program, the resulting discriminant function is used to distinguish among the different groups. Only images containing genuine particles as determined by the discriminant analysis are selected from the original unreduced micrograph. Optionally, one can window out the particles from the tilted micrograph simultaneously, as required by the random conical threedimensional reconstruction of molecules ${ }^{4}$.

This method works very well as long as the particles in the input image are isolated and not clustered together. The program was tested on the 70S E.coli ribosome data ${ }^{5}$. When using micrograph pairs with typically about 200-300 particles, it required approximately half an hour for the data preparation, 1.5-2.0 hours for the training and about 1.5 hours for the selection segments of the program, respectively. The precise time depends on the rejection rate. The times quoted here are all wall clock times on a VAX Workstation. For comparison, we mention here the average time taken for manual picking of particles from a similar pair which is about 6 hours. In typical reconstructions, one needs around 1500 2000 particles which would correspond to about ten micrograph pairs. Under such circumstances, this program is very effective since the training needs to be done only once for data of the same type, and the preparation and selection together take approximately a third of the time compared to manual picking ${ }^{6}$.

J. Frank and T. Wagenknecht, Uitramicroscopy. 12(1984)169.

M. van Heel, Uitramicroscopy. 8(1982)331.

G. Harauz and A. Fong-Lochovsky, Utramicroscopy. 31(1989)333

M. Rademacher, J. Electron Microsc. Tech. $9(1988) 359$.

P. Penczek, R.A. Grassucci and J. Frank, Uitramicroscopy. (1994, in press)

Supported, in part, by grant NIH 1R01GM29169 (to J.F.)

Reprinted from Proceedings of the 52nd Annual Meeting of the Microscopy Society of America.

\section{Light Element Peaks Revealed!}

\section{Oil Film on EDX Windows Removed:}

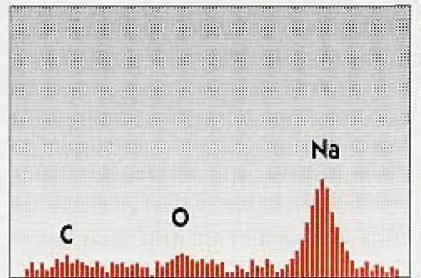

Oily Window

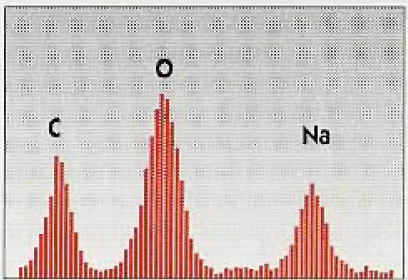

Clean Window
Oil build-up on EDX detector windows can ruin sensitivity for light element X-rays in SEMs. To stop oil condensation and keep the system clean, smart SEM users rely on the XEI Scientific SEM-CLEAN ${ }^{T M}$ system.

Result: Consistent light element X-ray results and contaminationfree pictures. The Nitrogen purge of the inexpensive SEM-CLEAN system actively cleans your electron microscope while you're away.

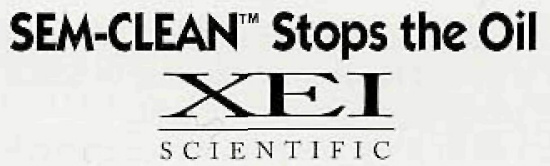

3124 Wessex Way, Redwood City, CA 94061 415-369-0133 • Fax 415-363-1659

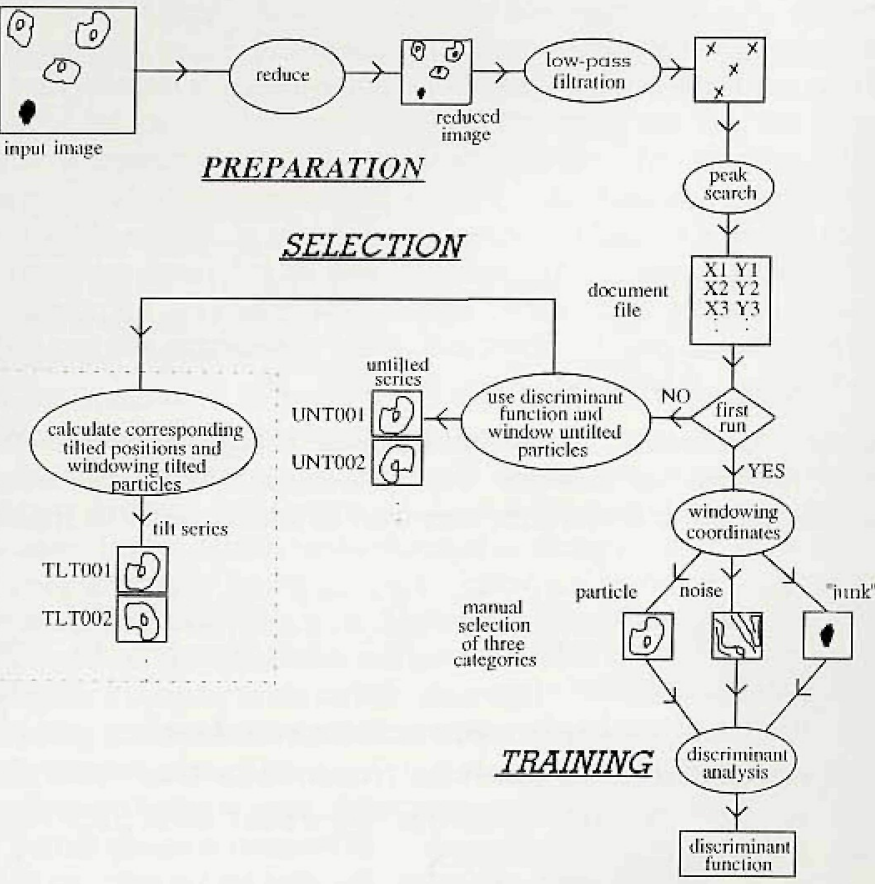

Fig. 1: Block diagram showing the sequence of operations in automatic particle selection The oval shapes refer to operations performed. The operations within the box drawn within the box drawn in dashed lines are optional 


\section{Simultaneous Multi Detector analysis without compromise.}

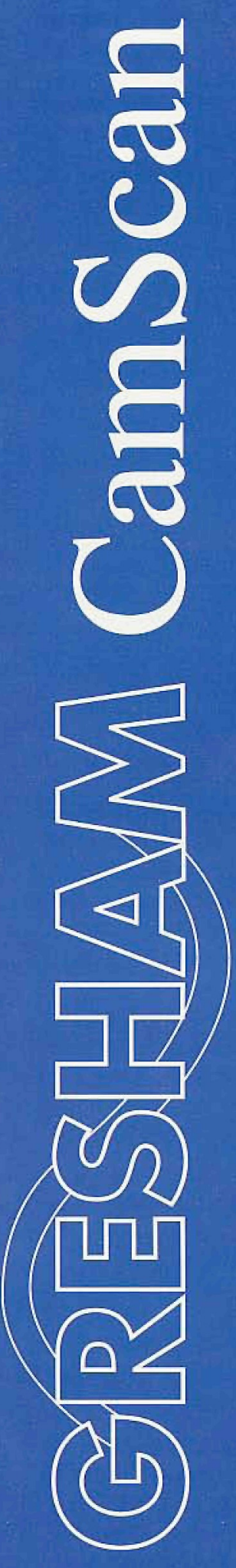

Quality of design

Quality of function

Quality of performance

New - from Gresham CamScan, the true analytical scanning electron microscope that gives you multiple and simultaneous detector capabilities without compromising the results of any individual technique.

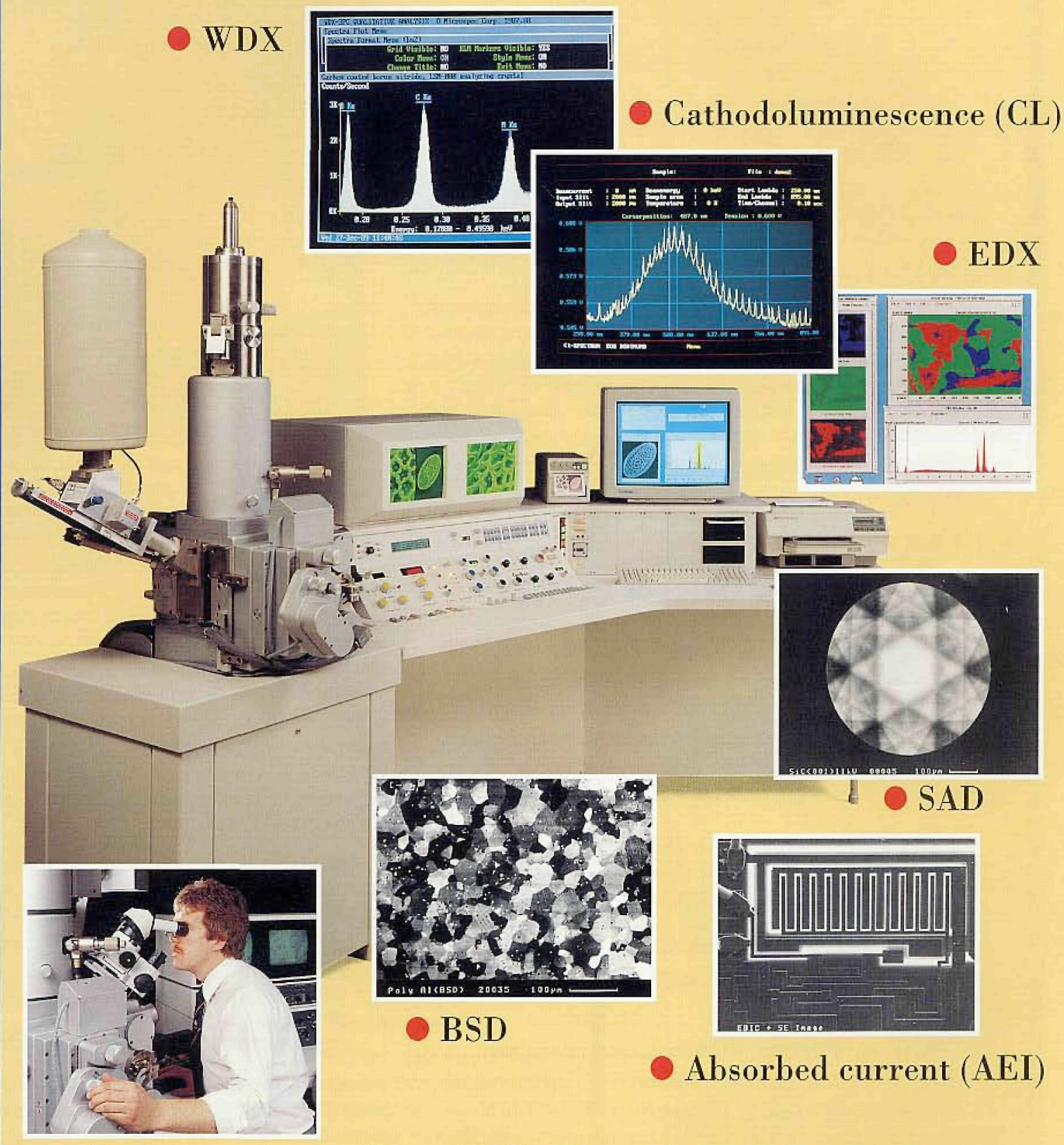

Optical

For a full information pack contact;

\section{(SRESTLAM}

Gresham-CamScan

Saxon Way, Bar Hill, Cambridge, CB3 8SL UK.

Tel: $+44(0) 1954780926$ Fax: +44 (0)1954 789829

\section{CamScan}

CamScan USA Inc.

508 Thomson Park Drive, Cranberry Township, PA 16066-6425 USA. Tel: (412) $772-7433$

Fax: (412) $772-7434$ 